\title{
Individual variability in functional connectivity predicts performance of a perceptual task
}

\author{
Antonello Baldassarre ${ }^{a, b, 1}$, Christopher M. Lewis ${ }^{a, b, c, 1}$, Giorgia Committeri ${ }^{a, b}$, Abraham Z. Snyder $^{d}$, \\ Gian Luca Romani ${ }^{a, b}$, and Maurizio Corbetta ${ }^{a, b, d, e, 2}$
}

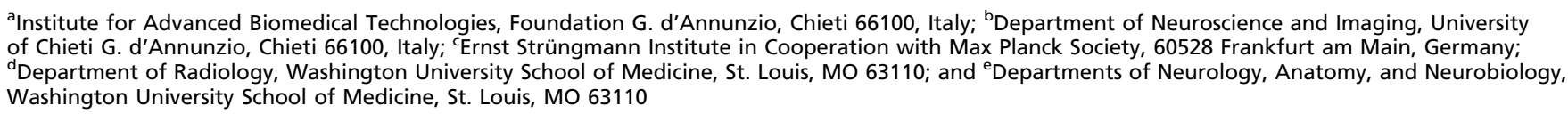

Edited by Michael Posner, University of Oregon, Eugene, OR, and approved December 30, 2011 (received for review August 15, 2011)

People differ in their ability to perform novel perceptual tasks, both during initial exposure and in the rate of improvement with practice. It is also known that regions of the brain recruited by particular tasks change their activity during learning. Here we investigate neural signals predictive of individual variability in performance. We used resting-state functional MRI to assess functional connectivity before training on a novel visual discrimination task. Subsequent task performance was related to functional connectivity measures within portions of visual cortex and between visual cortex and prefrontal association areas. Our results indicate that individual differences in performing novel perceptual tasks can be related to individual differences in spontaneous cortical activity.

predisposition | visual learning | spontaneous activity

$\mathbf{H}^{2}$ ealthy observers differ in their ability to perform a variety of visual tasks (1). Individuals also differ in their ability to improve with training (2-6), i.e., perceptual learning $(7,8)$. Initial performance and rate of learning tend to be inversely related $(3,9)$. Thus, individuals who perform better initially tend to exhibit slower improvement. Although the physiological correlates of perceptual learning have been well documented at the level of individual synapses $(10)$, neurons $(11,12)$, and largescale networks $(6,13-16)$, it is largely unknown whether the state of the brain before training influences future performance or the rate of acquisition of a novel task. Here we investigate the extent to which performance of a novel perceptual task can be predicted on the basis of physiological measures evaluated before training. Our measure of performance takes into account both early and late features of the psychophysical learning curve.

Intrinsic neural activity is temporally correlated within widely distributed networks that recapitulate the topography of task-related functional responses (17-21). Hence, resting-state functional connectivity offers a plausible neural correlate of behavioral predisposition to perform a novel task. Moreover, resting-state measures have been correlated with individual performance variability in several cognitive domains (22-26). However, to our knowledge, no study to date has shown that functional connectivity, measured before training, within cortical circuits later recruited by a novel task, is predictive of future performance.

In previous work (15), we showed that resting-state blood oxygenation level-dependent signal functional connectivity (FC) changes in task-relevant cortical networks after extensive practice on a novel orientation discrimination task. Critically, postlearning modulations in FC correlated with individual measures of improvement.

Here, we analyze the same dataset to test the hypothesis that FC in task-relevant circuits, measured before training, is predictive of subsequent performance.

\section{Results}

Behavior. Healthy observers ( $n=14$, seven male) were trained to report the presence/absence of a target shape (an inverted letter
T) (14) always presented in the lower left visual quadrant (15) (Fig. 1A, Methods, and SI Methods). Targets and distractors (letter Ts of different orientation) were presented together in a circular array at $5^{\circ}$ of eccentricity. The criterion for successful acquisition of the task was a normalized accuracy equal to or greater than $80 \%$ over 10 consecutive blocks of trials, with each block including 45 trials $(14,15,27)$ :

$$
\begin{aligned}
\text { Normalized accuracy }= & (\text { hits }[\%]+\text { correct rejections }[\%]) \\
& - \text { false alarms }[\%] / 1-\text { false alarms }[\%])
\end{aligned}
$$

On average, observers took approximately 5,600 trials or 118 blocks ( $\sim 4$ of $2-3 \mathrm{~h}$ practice per day) to reach the criterion (Fig. $1 B$ ). We observed a high degree of individual variability at the beginning of training. Accuracy on the first 10 blocks, the minimum number of blocks performed on the first day, was $41 \%$ with large interindividual variability (range, 13-69\%). Psychophysical performance curves were fit by using the following empirical two-parameter expression:

$$
a=a_{0}+s \log (k)
$$

where $a$ is accuracy, $k$ indexes block, $a_{0}$ is initial accuracy on the first block, and $s$ is a scaling parameter numerically equal to the initial slope. Fits of the analytic expression to the individual performance data were expressed in terms of variance explained $\left(r^{2}\right)$ : median $r^{2}$ was 0.68 , with a range of 0.29 to 0.93 (Fig. $1 B$ and Fig. S1). In addition to $a_{0}$ and $s$, we evaluated the number blocks needed to achieve the criterion (performance $\geq 80 \% ; k_{c}$ ). The three measures were correlated (SI Methods and Fig. S2) in a manner consistent with previous studies of perceptual learning $(3,9)$. Thus, subjects with high initial accuracy learned the task in fewer blocks but at a lower rate of improvement. Conversely, subjects with lower initial accuracy took longer to reach criterion but their rate of improvement was higher. Because of these relations and the relatively small size of the study group, it was not possible to derive independent measures of initial performance and rate of learning. To obtain individual quantitative indices of performance, $a_{0}, s$, and $k_{c}$ were entered into a principal component analysis (Fig. S3). The first component (PC1) explained 75\%

Author contributions: A.B., C.M.L., G.C., and M.C. designed research; A.B., C.M.L., and M.C. performed research; A.B., C.M.L., and A.Z.S. analyzed data; and A.B., C.M.L., G.C., A.Z.S., G.L.R., and M.C. wrote the paper.

The authors declare no conflict of interest.

This article is a PNAS Direct Submission.

See Commentary on page 3201.

${ }^{1}$ A.B. and C.M.L. equally contributed to this work.

${ }^{2}$ To whom correspondence should be addressed. E-mail: mau@npg.wustl.edu.

This article contains supporting information online at www.pnas.org/lookup/suppl/doi:10. 1073/pnas.1113148109/-/DCSupplemental. 

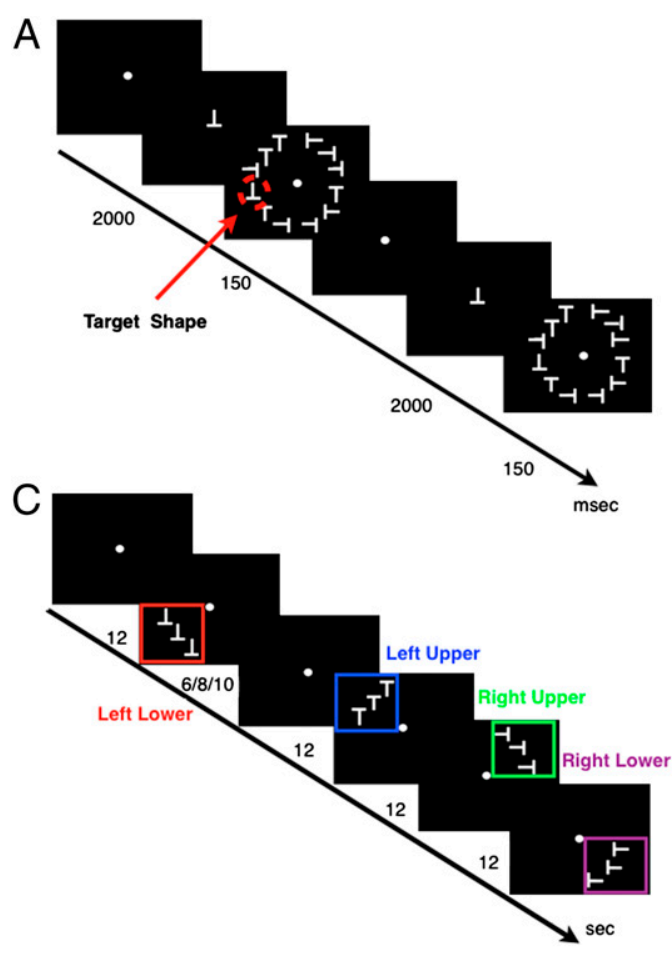
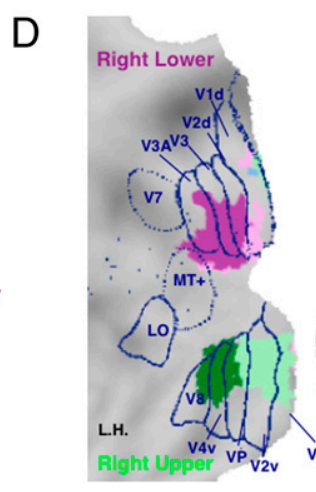
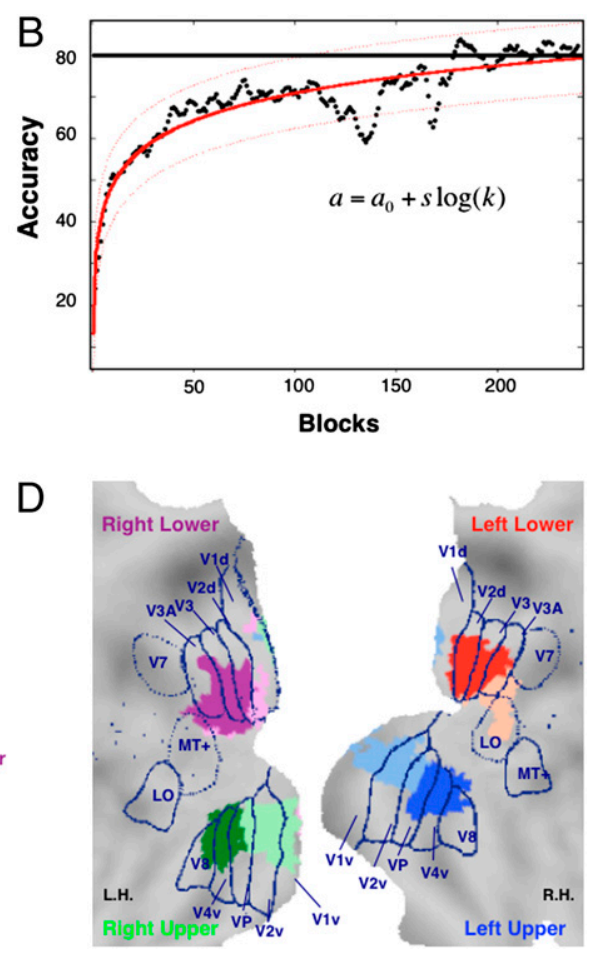

Fig. 1. Behavioral training, psychophysics results, visuotopic localizer, and ROls. (A) Experimental paradigm. (B) $x$ axis, number of blocks; $y$ axis, accuracy (i.e., percentage of correct response corrected for percentage of false alarms). Black dots display the group average performance block by block; solid red line indicates the psychophysical fitting model $a=a_{0}+s \log (k)$ with prediction bounds at $95 \%$ of confidence level (dotted lines). (C) Design of visuotopic localizer. Squares of different colors (not shown in real display) indicate a visual quadrant. $(D)$ Visual ROls/seeds. Eight visual regions (seeds) defined on the basis of the visuotopic localizer scan are displayed on the flattened representation of posterior occipital cortex using the PALS atlas (29). Blue lines are approximate borders between retinotopic visual areas based on a standard atlas (29) L.H., left hemisphere; R.H., right hemisphere. of the variance. The second component accounted for $15 \%$ of the variance, but its eigenvalue was less than 1 (scree plot in Fig. S3) and it was therefore not further considered (28). Accordingly, PC1 was used to compute individual measures of performance, which we here define as task fitness $(f)$ by using the following expression:

$$
f=\left[a_{0} s k_{c}\right] \cdot w
$$

where $w$ is the vector of factor weights (SI Methods). In the rest of the analysis, we use task fitness to examine the relationship between performance and pretraining resting-state FC.

Pretraining FC in Visual Cortex and Task Fitness. Resting-state functional MRI (fMRI) and visuotopic localizer fMRI were acquired 24 to $48 \mathrm{~h}$ before first exposure to the task (Methods and SI Methods). During the visuotopic localizer scans, subjects were asked to maintain central fixation while quarter-field stimulus arrays were passively presented in a blocked design (Fig. 1C). Regions of interest (ROIs) for the computation of FC were identified in the ventral and dorsal portions of visual cortex in each hemisphere. At the group level, two ROIs were identified in each quadrant as showing the strongest visuotopic localizer responses (e.g., in right dorsal cortex for left lower field stimulation) compared with the average response to stimuli in the other quadrants [group-level voxel-wise random-effect ANOVAs, multiple comparison corrected over the entire brain $(P<0.05)]$. These regions are shown in Fig. $1 D$ on a flattened representation of visual cortex in the Population Average Landmark and Surface (PALS) atlas (29) and labeled according to their location with respect to the probabilistic borders of visual areas in the same atlas (Table S1). In general, for each quarter-field representation in visual cortex, one ROI is "early" in the visual hierarchy (near/ at V1-V2), whereas the other is "intermediate" (near/at V4-V8 or V3A; Fig. $1 D$; Table S1 shows coordinates).

To examine the relationship between pretraining FC and the ability to perform the discrimination task, we computed grouplevel voxel-wise maps of the Pearson correlation coefficient between task fitness and the strength of FC for each visuotopic
ROI (defined as FC-PC1 correlation maps; Methods, SI Meth$o d s$, and Fig. S4). Fig. $2 A$ shows that the strength of FC between a representative ROI in right ventral visual cortex (near/at V1V2) and large swaths of ventral and dorsal peripheral visual cortex in both hemispheres is strongly correlated with task fitness (all voxels $\mathrm{Z}>2 ; P<0.05$, Monte Carlo corrected). Observers with stronger pretraining FC between visual regions displayed greater task fitness (Fig. 2B). This relationship was consistent across different ROIs in left and right visual cortex (Fig. S5). To quantify this consistency, a conjunction map was computed that shows the portions of visual cortex with behaviorally predictive FC across multiple ROIs (Fig. 2C). The most consistent regions encompassed both early and intermediate retinotopic areas, including a band outside the foveal region in the near periphery (based on the PALS borders).

To examine whether the regions exhibiting behaviorally significant pretraining FC coded for the stimuli, we quantified the percentage of voxels in the FC-PC1 conjunction map that overlapped with the regions in visual cortex selectively activated by the stimulus array (i.e., the sum of the quadrant maps). At a threshold of four of eight ROIs, $72 \%$ of the behaviorally predictive voxels from the FC-PC1 conjunction map fell within the borders of the region activated by the stimulus (Fig. $2 D$ ). This proportion increased to $86 \%$ when the threshold was increased to five of eight ROIs.

Computing pairwise correlations for all ROIs and calculating the correlation with task fitness confirmed these findings. The range of FC-PC1 correlations varied between an $r$ of 0.1 and an $r$ of $0.8 ; 13$ of 28 ( or $8 * 7 / 2$ ) possible ROI pairs showed a significant correlation with task fitness [false discovery rate (FDR), $q<0.05$ after random permutation test]. Thus, voxel-wise and regional analyses confirmed a significant relationship between task fitness and pretraining FC in portions of visual cortex activated by the visuotopic localizer stimuli. Fig. $3 A$ shows the group average strength of FC between ROI pairs arranged by visual quadrant (i.e., dorsal, ventral). Fig. $3 B$ shows behaviorally significant FC. Behaviorally predictive correlations (FC-PC1) were observed predominantly in heterotopic region pairs, i.e., region pairs in different quadrants within the same (e.g., left dorsal to ventral 

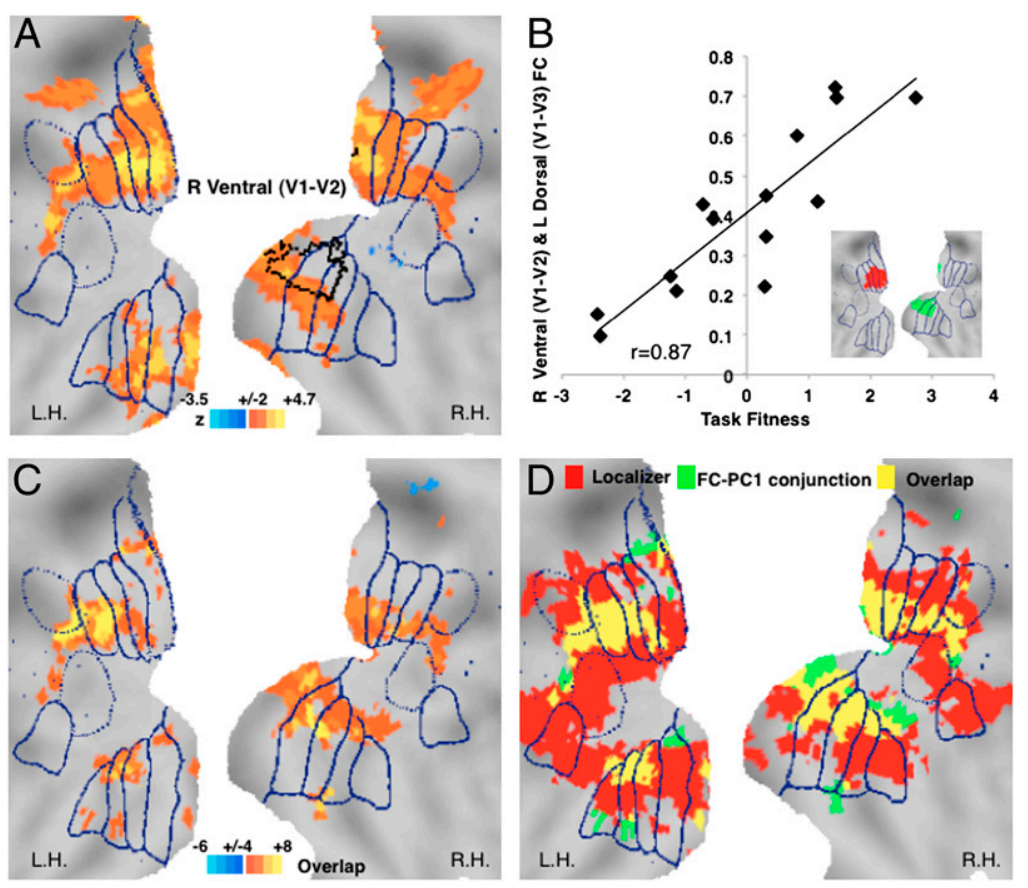

Fig. 2. Task fitness and pretraining $F C$ to/from visual cortex. (A) Voxel-wise FC-PC1 correlation map starting from a seed region in the right ventral visual seed (V1-V2; black border), corresponding to the left upper visual quadrant. The map is projected onto a flattened representation of the posterior occipital cortex using the PALS atlas (29). Color scale: yellow/ orange indicates positive correlation (Z-statistic of Pearson $r$ ) thresholded at $Z>2, P<0.05$, and Monte Carlo corrected. Blue color indicates negative correlation. Blue lines are the same as in Fig. 1D. L.H., left hemisphere; R.H., right hemisphere. $(B) x$ axis, task fitness, i.e., principal component scores of PC1; $y$ axis, FC (Fisher Z-transformed) between a right ventral visual seed V1-V2 (green, Inset; same as in A) and a left dorsal visual region (red) extracted from the FC-PC1 correlation map in $A$ (Talairach coordinates, $-06-96+08 ; 185$ voxels). Each diamond represents an observer. (C) Conjunction of eight voxelwise FC-PC1 correlation maps, one for each visual seed shown in Fig. $1 D$ (Table S1 provides coordinates). Color scale: yellow/ orange indicates overlap of positive correlations (range, 1-8); cyan/blue indicates overlap of negative correlations. $(D)$ Conjunction map between visuotopic localizer activations ( $Z$ statistic $>3, P<0.05$, Monte Carlo corrected; Methods; red) and FC-PC1 conjunction map thresholded four of eight (green). Overlapped voxels are in yellow.

cortex) or different hemispheres (e.g., left dorsal to right ventral cortex; Fig. $3 B$ and $C$ ), rather than homotopic region pairs (e.g., right dorsal to left dorsal cortex; Fig. $3 B$ and $D$ ) or local connectivity (e.g., right dorsal V1-V3 to right dorsal V3A-LO; permutation test on the entire correlation matrix, FDR $\mathrm{q}<0.05$; Fig. $3 B$ and $E$ ).

Pretraining FC Between Visual and Frontoparietal Regions and Task Fitness. Behaviorally predictive FC with visuotopic areas extended also to a small number of regions in higher-order frontal and posterior parietal cortex (Fig. S6 $A$ ). Fig. $4 A$ shows FC between a right dorsal visual ROI and left anterior insula, belonging to the "control network" $(30,31)$, which was negatively correlated with task fitness. Observers who performed better on the orientation discrimination task tended to have stronger negative correlation (i.e., antiphase coherence) between spontaneous activity in visual cortex and anterior insula (Fig. 4B). This result is representative of multiple visual ROIs (four of eight visual ROIs; Fig. 4C). Interestingly, this region overlaps with an insular region activated by the orientation discrimination task (Fig. $4 D$ and SI Methods). A similar pattern was detected in the right medial prefrontal cortex, a part of the default mode network (32, 33) (Fig. $4 E-H)$. Again, more negative FC corresponded to greater task fitness (Fig. $4 F$ ). This region overlaps with a larger medial prefrontal region deactivated by task performance (Fig. $4 H$ and SI Methods). Similar negative FC-PC1 correlations, i.e., more negative $\mathrm{FC}$ corresponding to better performance, were detected in other default mode regions, in left middle temporal cortex and right/left angular gyrus (Fig. S6 $A$ and Table S2). All regions in higher-order cortex that showed predictive FC with visual cortex overlapped regions recruited by the orientation discrimination task (Fig. S6 $B$ and $C$ ), albeit in a small proportion of the total extent of cortex recruited by the task.

Control Analyses: Auditory Cortex. To examine the modality specificity of these effects, and rule out the possibility that FC-performance correlations merely reflected a high level of FC in neighboring areas, a set of control analyses was run on primary and secondary auditory regions (SI Methods; Table S1 shows coordinates). The auditory regions were selected based on two criteria: anatomical location and task deactivation during the orientation discrimination task. In fact, primary and secondary auditory regions are typically deactivated during visual tasks (32). From the auditory regions, we computed baseline FC and FCPC1 correlation maps, which were compared with the visual regions. Both neighboring visual (Fig. $\mathrm{S} 7 A$ ) and auditory regions (Fig. S7C) showed strong FC. However, auditory regions (Fig. $\mathrm{S} 7 D$ ), in contrast to visual regions (Fig. S7B), did not show a predictive relationship with task fitness. We conclude that the behaviorally predictive pretraining FC is modality specific, and is not driven by local connectivity.

\section{Discussion}

We show that certain patterns of resting state $\mathrm{FC}$ within visual cortex, and between visual cortex and higher-order cortical regions, represent neural predictors of observer predisposition to perform a novel orientation discrimination task. Several previous studies have reported correlations between performance measures and fMRI FC (22-26). However, this study, as far as we are aware, is the first to demonstrate that FC, before any exposure, is predictive of performance and acquisition on a novel task. In addition, its topography coincides with the areas subsequently recruited by task performance.

Task Fitness: Initial Performance, Rate, and Duration of Learning. The behavioral component identified by the factor analysis (i.e., PC1) combined aspects of initial performance (i.e., predisposition), the rate of performance improvement, and the quantity of practice required to reach criterion. Our observers were highly variable in their initial performance, a finding concordant with previous studies of complex visual tasks (1) as well as perceptual learning $(2-4,6)$. Interestingly, task fitness was positively correlated with initial performance, and negatively correlated with the rate of learning and the number of blocks to criterion. Hence, subjects with high initial performance reached criterion earlier but at a slower rate, consistent with early reports on perceptual learning $(3,9)$. Our results therefore suggest that the state of the system at the beginning of training may influence the way the observers learn when the task requires extensive cortical processing. 
A

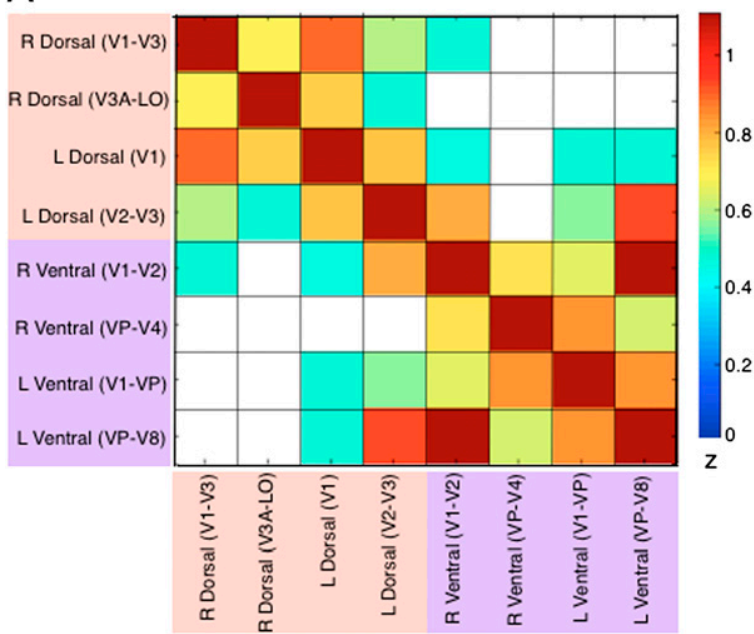

B

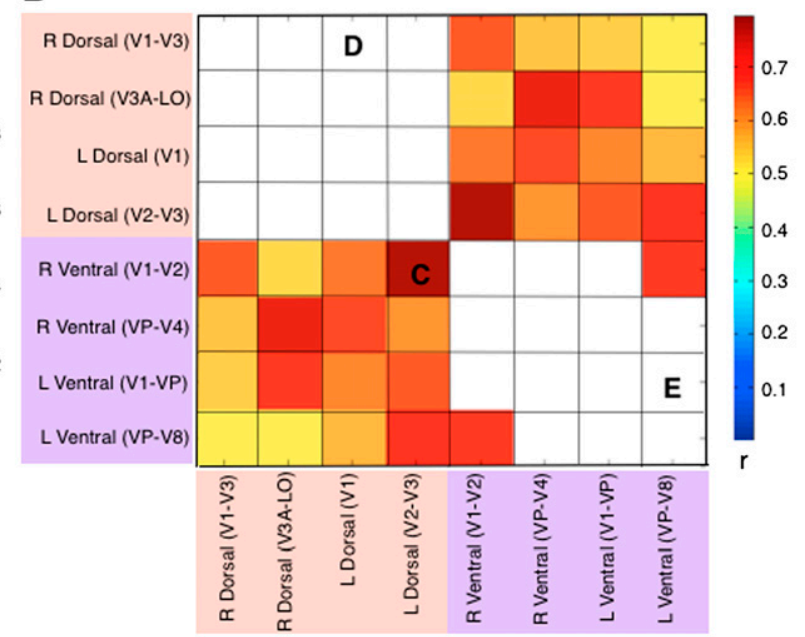

C

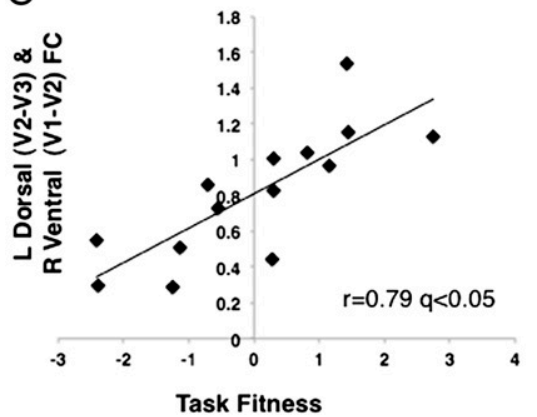

D

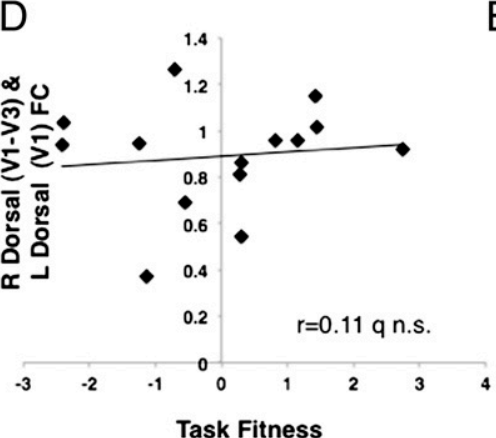

E

Fig. 3. Task fitness and pretraining FC within visual cortex. $(A)$ Correlation matrix (Fisher Z-transformed Pearson coefficient) of all ROI pairs in visual cortex. Yellow/orange color indicates positive correlations, white color indicates nonsignificant correlations (permutation test on the entire correlation matrix; FDR $q<0.05$ ). Dorsal visual regions are highlighted by light pink, ventral visual regions by light purple. (B) Correlation matrix (Pearson coefficient $r$ ) of PC1 and FC between all possible ROI pairs in visual cortex. Red/yellow cells indicate positive FC-PC1 correlations, white cells indicate nonsignificant correlations (permutation test on the entire correlation matrix; FDR $q<0.05)$. ( $C-E) x$ axis, task fitness, i.e., principal component scores of PC1; $y$ axis, FC (Fisher Z-transformed) between two heterotopic $(C)$, homotopic $(D)$, and neighboring $(E)$ visual regions. Each diamond represents an observer. For Pearson correlation coefficient, permutation test was performed on the entire correlation matrix (FDR $q<0.05$ ).

Predictive Intrinsic FC. Predictive FC was observed in region pairs including visual cortex as well as prefrontal and insular areas involved in cognitive control. All predictive regions were a subset of the cortical regions driven by the orientation discrimination task. Two patterns of FC were predictive of task fitness. In visual cortex, observers with stronger heterotopic functional connections, i.e., linking cortex representing dorsal and ventral quadrants within or between hemispheres, exhibited higher task fitness. In contrast, the strength of homotopic connections, i.e., linking dorsal or ventral quadrants across hemispheres, or of local connections, i.e., linking adjacent regions in visual cortex, was not predictive of performance. This result is noteworthy because local and homotopic FC typically is stronger than heterotopic $\mathrm{FC}(34,35)$.

A possible interpretation is that heterotopic connections linking different quadrants in visual cortex are more important for the dynamic reweighting of functional connections that occur in the course of learning. The orientation discrimination task required subjects to direct spatial attention to the left lower quadrant. Important processes for acquisition of the task include filtering of distracters at multiple unattended locations (36), as well as coding of the locus of attention by gradients of activity across spatial maps $(37,38)$. Hence, a high degree of coherence between stimulus-specific regions in visual cortex, before any experience, may facilitate the subsequent parsing of relevant from irrelevant information, and facilitate the reweighting of functional connections among different quadrants in visual cortex. At the end of learning, in agreement with this hypothesis, stronger responses to target shapes were recorded only in the trained visual quadrant, and FC was differentially modulated in trained and untrained quadrants (15).

The second predictive pattern of FC was an inverse correlation between spontaneous activity in visual cortex and regions of the default mode $(32,33)$ and control $(30)$ networks. This finding is also consistent with learning-related changes reported in our previous study (15), and in the work of Sigman et al. (14). We previously found that $\mathrm{FC}$ between unattended quadrants in visual cortex and default mode regions decreased (i.e., became less negative) after learning, and that these decrements correlated with measures of perceptual learning. Sigman et al. reported that decreases in task-evoked deactivation in the default mode network correlated with learning on the same task (14). Observers with stronger negative correlation between visual cortex and default mode regions at baseline may find it easier to filter out distracters at unattended locations early in training, which becomes less important as target selection becomes more automatic. This interpretation is consistent with a role of the default mode network in filtering out unattended stimuli, as suggested by other studies (39-41). Behaviorally significant negative correlations in FC between visual cortex and default mode regions have also been reported in relation to reading skills in children and adults, a competency closely related to orientation discrimination (25). 

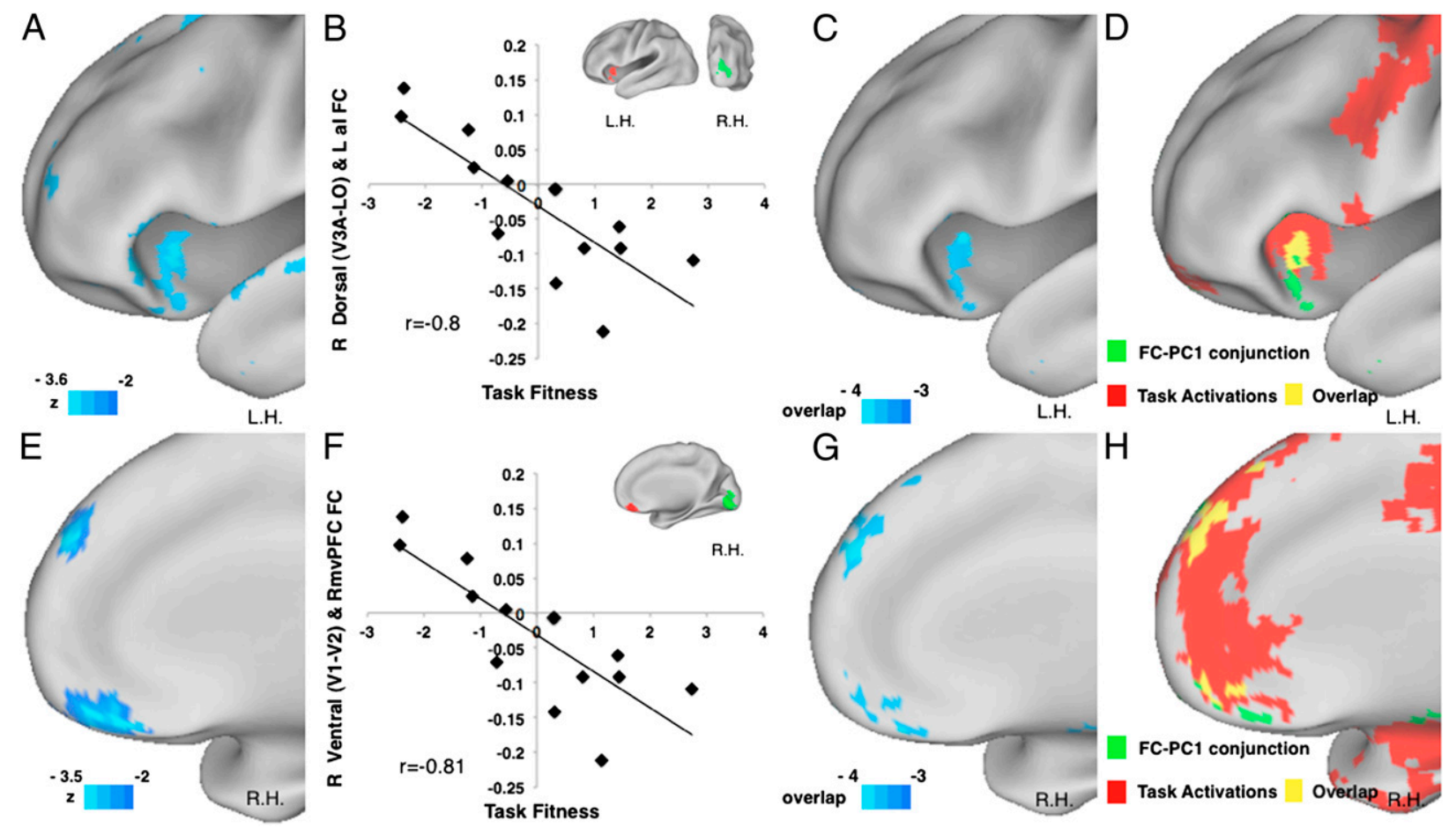

Fig. 4. Task fitness and pretraining FC to/from visual cortex and frontal regions. $(A)$ Lateral view of the voxel-wise FC-PC1 correlation map starting from a right dorsal visual seed (V3A-LO), corresponding to the left lower visual quadrant. Color scale is the same as in Fig. 2. L.H., left hemisphere. (B) $x$ axis, task fitness; $y$ axis, FC (Fisher Z-transformed) between a right dorsal visual seed V3A-LO (green, Inset; same as in A) and left anterior insula (Lal; red) extracted from the FC-PC1 correlation map in $A$ (Talairach coordinates, $-38+18-07 ; 171$ voxels). Each diamond represents an observer. (C) Conjunction map of FC-PC1 correlation maps from eight visual seeds. Color scale is the same as in Fig. 2. (D) Conjunction map between activation map of orientation discrimination task (trained plus untrained shape greater than fixation, Z-statistic $>3, P<0.05$, Monte Carlo corrected; Methods) and FC-PC1 conjunction map thresholded at negative three of eight (green). Overlapped voxels are in yellow. $(E)$ Medial view of the voxel-wise FC-PC1 correlation map starting from a right ventral visual seed (V1-V2), corresponding to the left upper visual quadrant. Color scale is the same as in $A$. R.H., right hemisphere. ( $F) x$ axis as in $B$; $y$ axis is FC (Fisher Z-transformed) between a right ventral visual seed V1-V2 (green, Inset) and a right ventral medial prefrontal cortex (RvmPFC; red) extracted from the FC-PC1 correlation map in $A$ (Talairach coordinates, $+04+38-18 ; 120$ voxels). Each diamond represents an observer. (G) Medial view of the same conjunction map in $B$, with the same color scale. $(H)$ Conjunction map between deactivation map of orientation discrimination task (trained plus untrained shape less than fixation, $Z$-statistic $>3$, $P<0.05$, Monte Carlo corrected; Methods) and FC-PC1 conjunction map thresholded negative three of eight (green). Overlapped voxels are in yellow.

Putative Underlying Mechanisms. One possible substrate for the predictive relationship between task fitness and FC is individual variability in structural connectivity. The strength of structural connections has been correlated with the strength of FC at the level of both large-scale networks (42) and local microcircuitry (43), and has also been shown to exhibit experience-dependent plasticity (44). However, in our study, regions exhibiting predictive FC (i.e., heterotopic connections in visual cortex, and visual, default, and control) showed weaker baseline FC. The logical inference would be that these areas are less well anatomically connected (42). Moreover, in primate studies, subdivisions of visual cortex with behaviorally predictive (i.e., heterotopic) functional coupling tend to have weaker anatomical connectivity than nonpredictive (i.e., homotopic) regions (45).

Another mechanism that could be related to our results is the recent observation that fMRI FC is related to slow cortical potentials and band-limited fluctuations of power in higher frequencies (46-48). These relatively slow fluctuations in neural excitability may facilitate synchronization of high-frequency activity through a variety of mechanisms $(49,50)$, and enable the coordination of task-relevant circuits. This could explain why observers with stronger FC within visual cortex, or between visual and other task-relevant areas in prefrontal and insular cortex, can recruit those regions more efficiently when performing a novel task.

We conclude that individual variability of FC within visual cortex, and between visual and higher-order regions, is related to the predisposition to perform a novel visual discrimination task.
These findings suggest a potential role of intrinsic brain activity as a neural predictor of perceptual skill acquisition. This result has general implications for the functional significance of spontaneous activity, and the neural bases of individual behavioral variability. In addition, our findings emphasize the importance of spontaneous activity, and the state of FC, as a possible "neural" prior for biasing task-evoked activity and behavior (51-53).

\section{Methods}

Participants. Healthy right-handed observers $(N=14)$ provided written informed consent approved by the Research Ethics Board of the University of Chieti.

Behavioral Training. Observers were instructed to attend to the left lower visua quadrant and report with a key press the presence/absence of a target shape (an inverted letter $\mathrm{T}$ ) in a briefly presented radial display of randomly oriented letter-T distracters. Central fixation was monitored with an eye tracker. Criterion for learning was 10 blocks of trials with accuracy of at least $80 \%$.

fMRI Scanning. Functional images (gradient-echo sequence, repetition time of $2.163 \mathrm{~s}$, echo time of $50 \mathrm{~ms}$, flip angle $90^{\circ}$, slice thickness of $8 \mathrm{~mm}, 3.75 \times 3.75$ $\mathrm{mm}$ in-plane resolution) were acquired during passive stimulation of each visual quadrant with the same display used for perceptual learning (i.e., localizer). Localizer scans were used to define ROIs/seeds for the FC analysis of resting-state data obtained before any exposure to the task.

Behavioral Score. Task fitness was defined as the first factor (i.e., PC1) of a principal component analysis on the parameters of a natural logarithmic function, plus the number of blocks to criterion, used to quantify observer learning curves. This component accounted for $75 \%$ of the behavioral 
variance and was correlated with initial performance, rate of learning, and number of blocks to criterion.

FC-PC1 Correlation. Voxel-wise or ROI pair FC-PC1 correlations were computed as the Pearson correlation coefficient $r$ between FC measures and task fitness (Results). FC was conventionally computed as the Pearson correlation between the time series extracted from a predefined ROI (e.g., left ventral visual cortex) and the rest of the brain (to obtain voxel-wise maps) or another ROI (to obtain ROI-ROI FC; SI Methods provides detailed information). Voxel-wise statistical significance in correlation maps was evaluated by first expressing the result as equi-probable $Z$ score maps, which were then corrected for multiple comparisons. Significance thresholds for ROI pair FC were

1. Halpern SD, Andrews TJ, Purves D (1999) Interindividual variation in human visua performance. J Cogn Neurosci 11:521-534.

2. Fahle M, Edelman S, Poggio T (1995) Fast perceptual learning in hyperacuity. Vision Res 35:3003-3013.

3. Fahle M, Henke-Fahle S (1996) Interobserver variance in perceptual performance and learning. Invest Ophthalmol Vis Sci 37:869-877.

4. Schmitt C, Kromeier M, Bach M, Kommerell G (2002) Interindividual variability of learning in stereoacuity. Graefes Arch Clin Exp Ophthalmol 240:704-709.

5. Fahle M (2004) Perceptual learning: A case for early selection. J Vis 4:879-890.

6. Mukai I, et al. (2007) Activations in visual and attention-related areas predict and correlate with the degree of perceptual learning. J Neurosci 27:11401-11411.

7. Gilbert CD, Sigman M, Crist RE (2001) The neural basis of perceptual learning. Neuron 31:681-697.

8. Sasaki $Y$, Nanez JE, Watanabe $T$ (2010) Advances in visual perceptual learning and plasticity. Nat Rev Neurosci 11:53-60.

9. Fahle M (1997) Specificity of learning curvature, orientation, and vernier discriminations. Vision Res 37:1885-1895.

10. Gilbert CD, Sigman M (2007) Brain states: Top-down influences in sensory processing Neuron 54:677-696.

11. Schoups A, Vogels R, Qian N, Orban G (2001) Practising orientation identification improves orientation coding in V1 neurons. Nature 412:549-553.

12. Raiguel S, Vogels R, Mysore SG, Orban GA (2006) Learning to see the difference specifically alters the most informative V4 neurons. J Neurosci 26:6589-6602.

13. Schwartz S, Maquet P, Frith C (2002) Neural correlates of perceptual learning: a functional MRI study of visual texture discrimination. Proc Natl Acad Sci USA 99: 17137-17142

14. Sigman M, et al. (2005) Top-down reorganization of activity in the visual pathway after learning a shape identification task. Neuron 46:823-835.

15. Lewis CM, Baldassarre A, Committeri G, Romani GL, Corbetta M (2009) Learning sculpts the spontaneous activity of the resting human brain. Proc Natl Acad Sci USA 106:17558-17563.

16. Li W, Piëch V, Gilbert CD (2004) Perceptual learning and top-down influences in primary visual cortex. Nat Neurosci 7:651-657.

17. Biswal B, Yetkin FZ, Haughton VM, Hyde JS (1995) Functional connectivity in the motor cortex of resting human brain using echo-planar MRI. Magn Reson Med 34 537-541.

18. Fox MD, et al. (2005) The human brain is intrinsically organized into dynamic, anticorrelated functional networks. Proc Natl Acad Sci USA 102:9673-9678.

19. Fox MD, Corbetta M, Snyder AZ, Vincent JL, Raichle ME (2006) Spontaneous neuronal activity distinguishes human dorsal and ventral attention systems. Proc Natl Acad SCi USA 103:10046-10051.

20. Vincent JL, et al. (2006) Coherent spontaneous activity identifies a hippocampal-parietal memory network. J Neurophysiol 96:3517-3531.

21. Greicius MD, Krasnow B, Reiss AL, Menon V (2003) Functional connectivity in the resting brain: a network analysis of the default mode hypothesis. Proc Natl Acad Sci USA 100:253-258

22. Hampson M, Driesen NR, Skudlarski P, Gore JC, Constable RT (2006) Brain connectivity related to working memory performance. J Neurosci 26:13338-13343.

23. Seeley WW, et al. (2007) Dissociable intrinsic connectivity networks for salience pro cessing and executive control. J Neurosci 27:2349-2356.

24. van den Heuvel MP, Stam CJ, Kahn RS, Hulshoff Pol HE (2009) Efficiency of functional brain networks and intellectual performance. J Neurosci 29:7619-7624.

25. Koyama MS, et al. (2011) Resting-state functional connectivity indexes reading competence in children and adults. I Neurosci 31:8617-8624.

26. Zhu Q, Zhang J, Luo YL, Dilks DD, Liu J (2011) Resting-state neural activity across faceselective cortical regions is behaviorally relevant. J Neurosci 31:10323-10330.

27. Sigman M, Gilbert CD (2000) Learning to find a shape. Nat Neurosci 3:264-269. computed by permutation simulations. Presently reported ROI pair results are FDR corrected with a $q$-value lower than 0.05

ACKNOWLEDGMENTS. We thank Drs. Annalisa Tosoni and Valentina Sebastiani for data collection; Dr. Francesco de Pasquale for discussion on data analysis; Dr. Gordon Shulman for reading and commenting on a draft of the manuscript; Dr. Mariano Sigman, one of the reviewers, for suggesting the principal component analysis on the behavioral data; and the other anonymous reviewer for constructive criticisms on the earlier draft. This work was supported by European Union (EU) Grants FP6-MEXC-CT-2004006783 (Ibsen) and FP7 200728 (Brain-Synch), National Institute of Mental Health Grant 1R01MH096482, National Institutes of Health Grant NS48013, and the Third PhD Internationalization Program of the Italian Ministry of University and Research.

28. Abdi H, Valentin D (2007) Multiple factor analysis (MFA). Encyclopedia of Measurement and Statistics, ed Salkind N (Sage Publications, Thousand Oaks, CA).

29. Van Essen DC (2005) A Population-Average, Landmark- and Surface-based (PALS) atlas of human cerebral cortex. Neuroimage 28:635-662.

30. Dosenbach NU, et al. (2006) A core system for the implementation of task sets. Neuron 50:799-812.

31. Dosenbach NU, et al. (2007) Distinct brain networks for adaptive and stable task control in humans. Proc Natl Acad Sci USA 104:11073-11078.

32. Shulman GL, et al. (1997) Common blood flow changes across visual tasks: II. Decreases in cerebral cortex. J Cogn Neurosci 9:648-663.

33. Raichle ME, et al. (2001) A default mode of brain function. Proc Natl Acad Sci USA 98: 676-682.

34. Vincent JL, et al. (2007) Intrinsic functional architecture in the anaesthetized monkey brain. Nature 447:83-86.

35. Stark DE, et al. (2008) Regional variation in interhemispheric coordination of intrinsic hemodynamic fluctuations. J Neurosci 28:13754-13764.

36. Gál V, et al. (2009) Learning to filter out visual distractors. Eur J Neurosci 29: 1723-1731.

37. Bisley JW, Goldberg ME (2003) Neuronal activity in the lateral intraparietal area and spatial attention. Science 299:81-86.

38. Sylvester CM, Shulman GL, Jack Al, Corbetta M (2007) Asymmetry of anticipatory activity in visual cortex predicts the locus of attention and perception. $J$ Neurosci 27 : 14424-14433.

39. Shulman GL, et al. (2003) Quantitative analysis of attention and detection signals during visual search. J Neurophysiol 90:3384-3397.

40. Shulman GL, Astafiev SV, McAvoy MP, d'Avossa G, Corbetta M (2007) Right TPJ deactivation during visual search: Functional significance and support for a filter hypothesis. Cereb Cortex 17:2625-2633.

41. Weissman DH, Woldorff MG (2005) Hemispheric asymmetries for different components of global/local attention occur in distinct temporo-parietal loci. Cereb Cortex 15:870-876.

42. Honey $\mathrm{CJ}$, et al. (2009) Predicting human resting-state functional connectivity from structural connectivity. Proc Natl Acad Sci USA 106:2035-2040.

43. $\mathrm{Ko} \mathrm{H}$, et al. (2011) Functional specificity of local synaptic connections in neocortical networks. Nature 473:87-91.

44. Johansen-Berg H (2007) Structural plasticity: Rewiring the brain. Curr Biol 17: R141-R144.

45. Gattas R, Sousa AP, Mishkin M, Ungerleider LG (1997) Cortical projections of area V2 in the macaque. Cereb Cortex 7:110-129.

46. Nir Y, et al. (2007) Coupling between neuronal firing rate, gamma LFP, and BOLD fMRI is related to interneuronal correlations. Curr Biol 17:1275-1285.

47. He BJ, Snyder AZ, Zempel JM, Smyth MD, Raichle ME (2008) Electrophysiological correlates of the brain's intrinsic large-scale functional architecture. Proc Natl Acad Sci USA 105:16039-16044.

48. de Pasquale F, et al. (2010) Temporal dynamics of spontaneous MEG activity in brain networks. Proc Natl Acad Sci USA 107:6040-6045.

49. Buzsáki G, Draguhn A (2004) Neuronal oscillations in cortical networks. Science 304: 1926-1929.

50. Fries $P$ (2005) A mechanism for cognitive dynamics: Neuronal communication through neuronal coherence. Trends Cogn Sci 9:474-480.

51. Arieli A, Sterkin A, Grinvald A, Aertsen A (1996) Dynamics of ongoing activity: Explanation of the large variability in evoked cortical responses. Science 273:1868-1871.

52. Kenet T, Bibitchkov D, Tsodyks M, Grinvald A, Arieli A (2003) Spontaneously emerging cortical representations of visual attributes. Nature 425:954-956.

53. Sadaghiani S, Hesselmann G, Friston KJ, Kleinschmidt A (2010) The relation of ongoing brain activity, evoked neural responses, and cognition. Front Syst Neurosci 4:20. 